\section{Truth Hits Everybody}

\section{Carlo Melloni*}

Center for Genital Reconstructive and Aesthetic Surgery, Palermo, Italy

\section{Editorial}

It is always a good thing promoting our work in the better way and even more often our patients ask us to show some "before and after" photos before undergoing surgery.

Someone use to say "if you don't take photos of your work is like haven't do anything" but, this time, I would like to focus on something that distinguish a good professional between others honesty.

Today it is more than obvious that we show our best results in our websites or social media but photoshop (a sophisticated image editing tool still used extensively by designers) has become a tool to make up miracles. In fact, someone use to spend more time sitting in front of a laptop and not in a surgical theater or using photoshop and mouse instead of a knife and stitches.

To put it bluntly, there are a lot of fake photos and photoshop is the tool used to create them.

In this editorial, I would like to drive your attention to some photoshop-addicted colleagues that mystify the "before and after" photos to attract patients and I'll try to explain how to recognize a fake.

Even more often our patients ask us to show some "before and after" photos before undergoing.

Photoshop was at one time a sophisticated image editing tool still used extensively by designers. Today it is more than obvious that we show our best results in our websites or social media but photoshop has become a tool to make up miracles and someone use to spend more time sitting in front of a laptop and not in surgical theater or using photoshop and mouse instead of a knife and stitches. To put it bluntly, there are a lot of fake photos and photoshop is the tool used to create them.

I would like to drive your attention to some photoshop-addicted colleagues that mystify the "before and after" photos to attract patients and trying to explain how to recognize a fake.

How do you tell if a photo has been tampered with? There are 5 simple rules to follow and on which pay attention.

1. Visual inspection: You might think that photoshop scam artists might perfect their game but there are often visual tell-tale signs that a photo has been manipulated. Even famous print magazines have come under fire for such blatant fake images. This proves one thing; if you know what to look for, a simple visual inspection will tell you a lot about how "real" a photo is.

2. Loss in quality: Consider this; phone cameras are ridiculously powerful today, they come with a flash, a night mode, and other features. How bad can a photo really get? Areas that have been photoshopped will appear in lower quality most times.

3. Pay attention to details: pigmented lesions, scars, striae...it is a good way to understand if the patient shown in the post-op pictures is the same of the pre-op. Same thing if you look at the hair. Time passes and hair grows. Does the patient cut his/her hair after surgery. Of course if the patient shows exactly the same position, same t-shirt, same wrinkles in the same position and
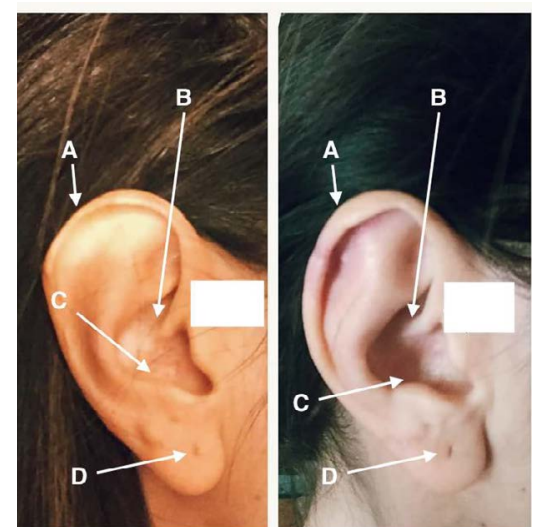

Figure 1: Pre-op and post-op of an otoplasty. (A) Auricular shape of the helix (B) Crus of helix (C) Antitragus (D) and earring hole are different. The signature of the surgeon has been masked by a white square.

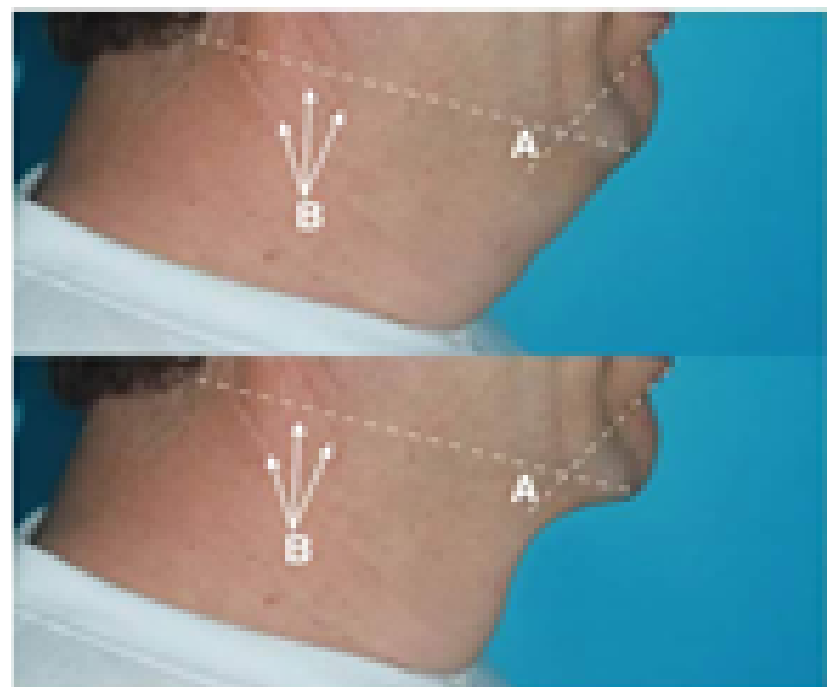

Figure 2: Pre-op and post-op of a neck lifting. Absence of edema and ecchymosis reveal a long term followup. The position of chin (A) and the lenght of the hair are the same, as well as the wrinkles (B). The patient also wear the same t-shirt. All of these are strongly suspicious of a photoshop fake.

${ }^{*}$ Corresponding author: Carlo Melloni, Gender Team, Center for Genital Reconstructive and Aesthetic Surgery,Via Sardegna, Palermo, Italy, Tel: 091528976; E-mail: studiomedico.melloni@gmail.com

Received January 11, 2017; Accepted January 13, 2017; Published January 21 2017

Citation: Melloni C (2017) Truth Hits Everybody. Reconstructive Surgery and Anaplastology 6: e118. doi: 10.4172/2161-1173.1000e118

Copyright: $\odot 2017$ Melloni C. This is an open-access article distributed under the terms of the Creative Commons Attribution License, which permits unrestricted use, distribution, and reproduction in any medium, provided the original author and source are credited. 

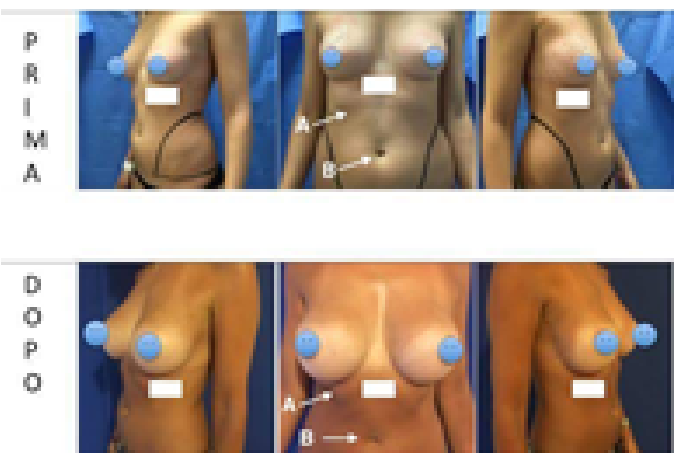

Figure 3: Pre-op and post-op of a breast lipofilling. The patient is tanned, quite strange after a surgery. The ribs shape (A) and navel $(B)$ are different. We don't think is the same patient. The signature of the surgeon has been masked by a white square. same hair lenght (Figure 2) probably it is a fake.

4. Look at the tan: It is pretty wierd that a patient underwent surgery in october is tanned in april (6 months post-op). Does he/she had a carribean trip during the postoperative period?

5. Look at the shapes: If you're looking on photos of a breast augmentation or an otoplasty or a abdominoplasty do not focus only at that level...try to look at the whole patient; see his/her shapes: shoulders, chest, navel, shapes of auricular cartilages (Figures 1,3).

Good ethics is a fundamental in any profession. It is an important part of the success in professions and private life. For a medical professional, the adherence to high ethical standards is mandatory but, unfortunately, the pursuit of business goals can be too much attractive for someone and several factors may encourage one to adopt unethical behaviour. It's fundamental to distinguish what's morally good and bad in order to follow the rules and code of professional conduct [1,2].

Good ethics will lead us to maintain our honest image. It will enable us to refrain from such activities that may discredit to our profession. Thus, adhesion to good ethics is to let our conscience be our guide at all times.

As The Police said in 1979... "Truth hits everybody".

\section{References}

1. Mansfield SJ, Morrison SG, Stephens OH, Bonning MA, Wang SH, et al. (2011) Social media and the medical profession. Med J Aust 194: 642-644.

2. F Siddiqui (2014) The importance of ethics in your professional life. 